\title{
Research Article \\ Compact Support FDK Kernel Reconstruction Model Base on Approximate Inverse
}

\author{
Jun Feng, ${ }^{1,2}$ Jian-Zhou Zhang, ${ }^{1}$ and Bin Zhou $^{3}$ \\ ${ }^{1}$ College of Computer, Sichuan University, Chengdu 610065, China \\ ${ }^{2}$ College of Management Science, Chengdu University of Technology, Chengdu 610059, China \\ ${ }^{3}$ College of Science, Xi'an University of Science and Technology, Xi'an 710054, China
}

Correspondence should be addressed to Jun Feng, fnjun@163.com

Received 31 October 2011; Accepted 22 November 2011

Academic Editor: P. Liatsis

Copyright ( $) 2012$ Jun Feng et al. This is an open access article distributed under the Creative Commons Attribution License, which permits unrestricted use, distribution, and reproduction in any medium, provided the original work is properly cited.

A novel CT reconstruction model is proposed, and the reconstruction is completed by this kernel-based method. The reconstruction kernel can be obtained by combining the approximate inverse method with the FDK algorithm. The computation of the kernel is moderate, and the reconstruction results can be improved by introducing the compact support version of the kernel. The efficiency and the accuracy are shown in the numerical experiments.

\section{Introduction}

In the last years, the approximate inverse method [1-3] is proposed and provides a general strategy for inverse problem. In computed tomography field, the approximate inverse is to process the measured data on the precomputed kernel to get an approximate reconstruction result. Recently, Louis et al. have described the relation between the X-ray and the Radon transform with the formula of Grangeat [4] by some proper operators and derived the kernel of Radon transform for the easy-to-implement circular scanning trajectory [5].

At present, image reconstruction from $X$-ray cone-beam projections collected along a single circular source trajectory is commonly done with the FDK reconstruction method despite of the drawback that the FDK method is inaccurate [4,6]. Due to its highly computational efficiency, many modifications for the FDK method have been proposed [7-9], but many of them are concentrated on improving the trajectory to fulfill the data-sufficient condition.

As the integral operators in most tomography problems are compact operators acting on suitable infinite dimensional Hilbert spaces, their inverse operators are not continuous, 
which would amplify the unavoidable data errors. When a reconstruction work is processing, the demand for highest possible accuracy and the necessary damping of the influence of the unavoidable data errors should be balanced [5]. Compared with the exact reconstruction algorithms, approximate algorithms can provide more flexible tradeoff between the image quality and computational efficiency. Thus, the FDK reconstruction method is highlighted to realize the approximate inversion for circular trajectory.

In this paper, a novel CT reconstruction model is proposed based on the approximate inverse where the kernel of the FDK method is derived and is used to complete the reconstruction. With the classical FDK method, the coordinate of the reconstruction point is first transformed from the globe Cartesian coordinate system to the detector coordinate system. It means that the reconstruction point is combined with the measured projection directly, and, furthermore, the invariance and symmetries of the coordinate system are preserved. Then the reconstruction kernel of the FDK method can be derived by defining some operators while the simplicity is preserved. In some ways, it presents a modification for the FDK method, and it is also a novel realization of the approximate inverse method. With the FDK kernel, the reconstruction can be completed efficiently. However, imposed ring artifacts arise around the area where the density is high.

Ring artifacts mainly arise in a third-generation CT system. They are caused by errors in a single or multiple channels over an extended range of views. It can be found that even a little projection errors can cause perceivable ring artifacts in the image. An error in an isolated view is mapped to a streak by the backprojection process. If the same error is persistent over a range of views, the streaks are blended, and an arc is generated [10].

From the figure of the FDK kernel, it can be observed that many minute oscillations exist at the edge of the kernel, and the FDK kernel has infinite support set. In consideration of the approximate inverse method, the oscillations present the contribution errors in multiple channels. As the shift-invariant property of FDK kernel is utilized in the back-projection process, and when the projections in the whole circle are considered, these errors generate the ring artifacts. Thus, in order to eliminate the ring artifacts, we update the reconstruction model by truncating the kernel with proper radiuses to make the kernel compact. For a given radius, the values of the points far from the center are set as zero, and thus the errors are set as zero. Reconstruction results show that the compact support FDK kernel is advantageous to reduce the ring artifacts and enhance the edge clarity.

The remainder of this article is organized as follows. In Section 2, the approximate inverse method and the kernel of the Radon transform are briefly introduced. In Section 3, the FDK algorithm is combined with the approximate inverse, and the FDK kernel is derived. Then the kernel is truncated to be compact and the compact kernel-based reconstruction model is introduced. And at last some properties of the FDK kernel are discussed. Experiments results and conclusion are presented in Sections 4 and 5.

\section{Approximate Inverse Method and Radon Kernel Reconstruction Model}

\subsection{Approximate Inverse Method}

In this section, the approximate inverse method is briefly introduced. For more details, we refer to $[1-3,5]$. 
Let $A: X \rightarrow Y$ be a compact operator between real Hilbert spaces $X$ and $Y$. The approximate inverse means a solution operator $S_{\gamma}$, which maps the data $g$ to $f_{\gamma}(x)$, a stable approximation of the solution $f(x)$ of the ill-posed problem

$$
\text { Af }=g \text {, }
$$

as follows:

$$
f_{\gamma}(x)=S_{\gamma} g(x)
$$

The approximation of $f_{\gamma}(x)$ is determined with a mollifier $e_{\gamma}(x, \cdot)$,

$$
f_{\gamma}(x)=\left\langle f, e_{\gamma}(x, \cdot)\right\rangle_{X^{\prime}}
$$

where the mollifier $e_{\gamma}(x, \cdot)$ is an approximation of the delta function $\delta_{x}(\cdot)$, for example the Gaussian function. And the parameter $\gamma$ is a regularization parameter.

With the auxiliary function

$$
A^{*} \Psi_{\gamma}(x, \cdot)=e_{\gamma}(x, \cdot)
$$

we can get the following:

$$
\begin{aligned}
f_{\gamma}(x) & =\left\langle f, e_{\gamma}(x, \cdot)\right\rangle_{X}=\left\langle f, A^{*} \Psi_{\gamma}(x, \cdot)\right\rangle_{X} \\
& =\left\langle A f, \Psi_{\gamma}(x, \cdot)\right\rangle_{Y}=\left\langle g, \Psi_{\gamma}(x, \cdot)\right\rangle_{Y} \\
& =: S_{\gamma} g(x),
\end{aligned}
$$

where $A^{*}$ is the adjoint operator of $A$. The solution of the auxiliary function: $\Psi_{\gamma}(x, \cdot)$ is called the reconstruction kernel, and the measured data can be directly processed with the kernel to get the reconstruction results.

\subsection{Radon Kernel Reconstruction Model}

In [5], Louis et al. gave the reconstruction kernel based on the Radon transform with the formula of Grangeat.

In circular tomography, let the condition of Tuy-Kirillove be fulfilled, the inversion of the 3D Radon transform is given as

$$
f=\frac{1}{8 \pi^{2}} D^{*} T M_{\Gamma} T D f
$$

hence, we can get the solution of the auxiliary function $D^{*} \Psi_{\gamma}=e_{\gamma}$ as

$$
\Psi_{\gamma}=\frac{1}{8 \pi^{2}} T M_{\Gamma} T D e_{\gamma}
$$


and the $\Psi_{\gamma}$ is the reconstruction kernel, where

$$
D f(a, \theta)=\int_{0}^{\infty} f(a+t \theta) \mathrm{d} t
$$

is the cone-beam transform of $f \in L_{2}(R)$, and its adjoint operator is

$$
D^{*} g(r)=\int_{\Gamma}\|r-a\|^{-2} g\left(a, \frac{r-a}{\|r-a\|}\right) \mathrm{d} a .
$$

The integral operator $\operatorname{Tg}(\omega)$ and multiplication operator $M_{\Gamma} h(a, \theta)$ are defined as

$$
\begin{gathered}
\operatorname{Tg}(\omega)=\int_{S^{2}} g(\theta) \delta(\langle\theta, \omega\rangle) \mathrm{d} \theta, \\
M_{\Gamma} h(a, \theta)=|\langle\dot{a}, \omega\rangle| m(\omega,\langle a, \omega\rangle) h(\omega),
\end{gathered}
$$

which are defined from the inverse of the 3D Radon transform

$$
f(r)=-\left.\frac{1}{8 \pi^{2}} \int_{S^{2}} \frac{\partial^{2}}{\partial s^{2}} R f(\omega, s)\right|_{s=\langle x, \omega\rangle} \mathrm{d} a,
$$

with the formula of Grangeat [4]

$$
\left.\frac{\partial^{2}}{\partial s^{2}} R f(\omega, s)\right|_{s=\langle x, \omega\rangle}=-\int_{S^{2}} D f(a, \theta) \delta^{\prime}(\langle\theta, \omega\rangle) \mathrm{d} \theta
$$

Let $n(\omega, s)$ denote the Crofton symbol, and setting $m=1 / n$ : (2.11) can be expressed as follows,

$$
\begin{aligned}
f(r)= & \frac{1}{8 \pi^{2}} \int_{\Gamma} \frac{1}{\|r-a\|^{2}} \iint_{S^{2}} D f(a, \theta) \delta^{\prime}(\langle\theta, \omega\rangle) \mathrm{d} \theta \\
& \times \delta^{\prime}\left(\left\langle\frac{r-a}{\|r-a\|^{2}}, \omega\right\rangle\right)|\langle\dot{a}, \omega\rangle| m(\omega,\langle a, \omega\rangle) \mathrm{d} a \mathrm{~d} \omega .
\end{aligned}
$$

With operators above, the inverse formula of the 3D Radon transform shown as (2.11) can be expressed as the operator equation as (2.6), and the reconstruction kernel $\Psi_{\gamma}$ is derived as (2.7).

As we know that the circular trajectory could not fulfill the sufficient condition, only approximate results are possible. At present, FDK reconstruction method is the most popular method for circular reconstruction. In next section, we concentrate on deriving the FDK kernel from the well-known FDK method and then proposed the compact support FDK kernel reconstruction model. 


\section{Compact Support FDK Kernel Reconstruction Model}

\subsection{FDK Reconstruction Method}

The 3D FDK reconstruction method is a heuristic development of the 2D Radon transform [6]. From the measured projection data along the intersection of the detector plane and the midplane, the density at points that lie in the midplane can be calculated. Then for a tilted plane which intersect the detector plane along a line parallel to the midplane, it is treated as the midplane of another tilted arrangement CT system. Making the corrections of the rotation and the distance between the actual midplane and the tilted plane, the density at the point $r$ in the tilted plane is the sum of the increment contributions from all planes that pass through $r$. The FDK reconstruction method can be expressed as follows:

$$
\begin{aligned}
f_{\mathrm{FDK}}(r)= & \frac{1}{4 \pi^{2}} \int_{0}^{2 \pi} \mathrm{d} \beta \frac{d^{2}}{(d+r \cdot \widehat{x})^{2}} \int_{0}^{+\infty} \omega \mathrm{d} \omega \int_{-\infty}^{+\infty} \mathrm{d} Y \\
& \times \frac{d}{\sqrt{d^{2}+Y^{2}+Z^{2}}} D_{\beta}(Y, Z) \exp \left[i w\left(\frac{d r \cdot \hat{y}}{d+r \cdot \widehat{x}}\right)-Y\right],
\end{aligned}
$$

where $\hat{x}=\overrightarrow{A O}, \hat{y}=\overrightarrow{A O}$ and the axis of rotation $\widehat{z}$ direction composed the local Cartesian coordinate system, $O$ is the origin, $A$ is the source point, $d$ is the distance from the source point $A$ to the hypothetic detector which contains the rotation axis and is parallel to the real detector, $(Y, Z)$ is the coordinate of the hypothetic detector plane, $D_{\beta}(Y, Z)$ denotes the projection acquired at the point $(Y, Z)$, and $\beta$ denotes the rotate angle of the source $A$.

\subsection{FDK Kernel Reconstruction Model}

Let $a \in \Gamma$ denotes the position of the source point $A$ on the circular trajectory $\Gamma$, given a fixed reconstruction point $r(x, y, z)$, where $x, y, z$ is the globe coordinate of the point. Then on the local Cartesian coordinate system of $\langle\widehat{x}, \widehat{y}, \widehat{z}\rangle$, the square of distance from the source $A$ to the reconstruction point $r(x, y, z)$ is

$$
\|r-a\|^{2}=(d+r \cdot \widehat{x})^{2}+(r \cdot \widehat{y})^{2}+(r \cdot \widehat{z})^{2},
$$

then (3.1) is revised into

$$
\begin{aligned}
f_{\mathrm{FDK}}(r)= & \frac{1}{4 \pi^{2}} \int_{0}^{2 \pi} \mathrm{d} \beta\|r-a\|^{-2}\left[(d+r \cdot \widehat{x})^{2}+(r \cdot \widehat{y})^{2}+(r \cdot \widehat{z})^{2}\right] \frac{d^{2}}{(d+r \cdot \widehat{x})^{2}} \\
& \times \int_{0}^{+\infty} \omega \mathrm{d} \omega \int_{-\infty}^{+\infty} \mathrm{d} Y \frac{d}{\sqrt{d^{2}+Y^{2}+Z^{2}}} D_{\beta}(Y, Z) \exp \left[i \omega\left(\frac{d r \cdot \hat{y}}{d+r \cdot \widehat{x}}\right)-Y\right] \\
= & \frac{1}{4 \pi^{2}} \int_{0}^{2 \pi} \mathrm{d} \beta\|r-a\|^{-2}\left[d^{2}+\left(\frac{d r \cdot \widehat{y}}{d+r \cdot \hat{y}}\right)^{2}+\left(\frac{d r \cdot \widehat{z}}{d+r \cdot \widehat{z}}\right)^{2}\right] \\
& \times \int_{0}^{+\infty} \omega \mathrm{d} \omega \int_{-\infty}^{+\infty} \mathrm{d} Y \frac{d}{\sqrt{d^{2}+Y^{2}+Z^{2}}} D_{\beta}(Y, Z) \exp \left[i \omega\left(\frac{d r \cdot \widehat{y}}{d+r \cdot \widehat{x}}\right)-Y\right] .
\end{aligned}
$$


Let

$$
y^{\prime}=\frac{d r \cdot \widehat{y}}{d+r \cdot \widehat{x}^{\prime}}, \quad z^{\prime}=\frac{d r \cdot \widehat{z}}{d+r \cdot \widehat{x}}
$$

where $y^{\prime}, z^{\prime}$ are the coordinates of the intersection point on the hypothetic detector of the line from the source to the reconstruction point: $\overrightarrow{a r}$. Then (3.3) above can be expressed as

$$
\begin{aligned}
f_{\mathrm{FDK}}(r)= & \int_{0}^{2 \pi} \mathrm{d} \beta\|r-a\|^{-2} \frac{1}{4 \pi^{2}}\left[d^{2}+y^{\prime 2}+z^{\prime 2}\right] \\
& \times \int_{-\infty}^{+\infty} \mathrm{d} Y \frac{d}{\sqrt{d^{2}+Y^{2}+Z^{2}}} \int_{0}^{+\infty} \omega \mathrm{d} \omega \exp \left[i \omega y^{\prime}-Y\right] D_{\beta}(Y, Z) .
\end{aligned}
$$

Define three new operators:

$$
\begin{gathered}
M(r, a, \theta)=d^{2}+y^{\prime 2}+z^{\prime 2}, \\
H(r, a)=\int_{0}^{+\infty} \omega \mathrm{d} \omega \exp [i \omega y] \\
W(r, a, \theta)=\frac{d}{\sqrt{d^{2}+Y^{2}+Z^{2}}}
\end{gathered}
$$

where the unit vector $\theta \in S^{2}$ denotes the direction of the projection. With the cone-beam transform as (2.8) and its adjoint operator as (2.9), the FDK reconstruction method can be denoted as follows:

$$
f_{\mathrm{FDK}}=\frac{1}{4 \pi^{2}} D^{*} M H * W D f,
$$

and the inverse of the projection is given as

$$
D^{-1}=\frac{1}{4 \pi^{2}} D^{*} M H * W .
$$

According to the auxiliary problem $D^{*} \Psi_{\gamma}(x, \cdot)=e_{\gamma}(x, \cdot)$, the kernel based on the FDK reconstruction method is derived,

$$
\Psi_{\mathrm{FDK}}(r, a, \theta)=\frac{1}{4 \pi^{2}} M H * W D e_{r} .
$$

Thus for a suitable mollifier $e_{\gamma}(x, y)$, a novel reconstruction kernel can be obtained. In this work, we choose a translational and rotational invariant function, the Gaussian function as the mollifier

$$
e_{\gamma}(x, y)=(2 \pi)^{-3 / 2} \frac{1}{\gamma^{3}} e^{-\|x-y\|^{2} / 2 \gamma^{2}} .
$$




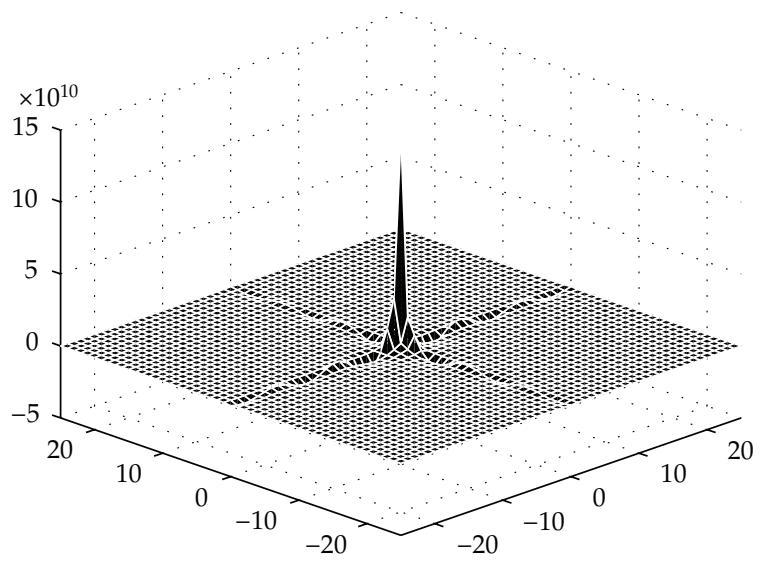

Figure 1: Kernel of the FDK method with the CT geometry in Table 1.

Table 1: Parameters of Measurement.

\begin{tabular}{lcc}
\hline & Measurement parameters & \\
\hline Detector & & $255 \times 255$ \\
Projections & 180 \\
Source-Detector & $\sim 100 \mathrm{~cm}$ \\
Source-Object & $\sim 87.3 \mathrm{~cm}$ \\
\hline & & \\
\hline Reconstruction grid & Reconstruction parameters & $255 \times 255$ \\
$\gamma$ & & $0.00165 \mathrm{~cm}$ \\
\hline
\end{tabular}

Then according to the approximate inverse method, the projections can be processed on the FDK kernel for reconstruction. Then we obtain the FDK kernel reconstruction model as:

$$
f_{Y}(x)=\left\langle g, \Psi_{\mathrm{FDK}}(x)\right\rangle
$$

The FDK kernel is computed in numerical experiments, and the shape of the original FDK kernel is shown in Figure 1.

\subsection{Compact Support FDK Kernel and the Reconstruction Model}

With the FDK kernel reconstruction model, we reconstruct the Shepp-Logan phantom and the Turbell clock phantom in numerical experiments. Reconstruction results are shown in Figures 2(c), 4(c), and 5(c). According to these results, we can see that the results based on the original FDK kernel have ring artifacts. Beside the ring artifacts, the results have homogenous density on the whole.

As we all know, the FDK reconstruction method is a filtered back-projection reconstruction method. From the formula of the kernel as (3.9), the FDK kernel is a convolution type operator. In computing the FDK kernel, as a result of the approximate inverse method, shown in Figure 1, many minute oscillations exist at the edge which make the support set of 


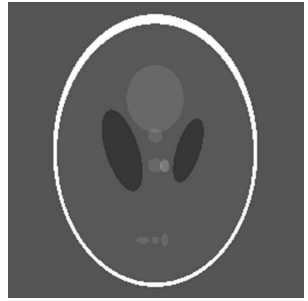

(a)

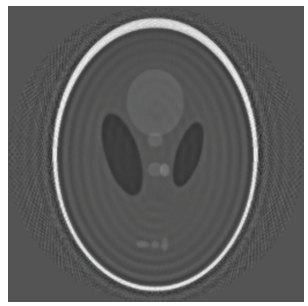

(d)

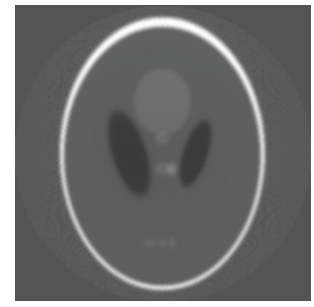

(b)

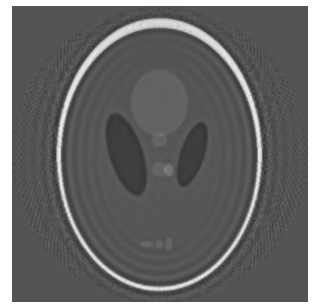

(e)

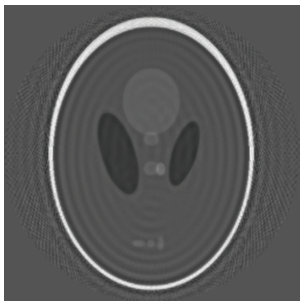

(c)

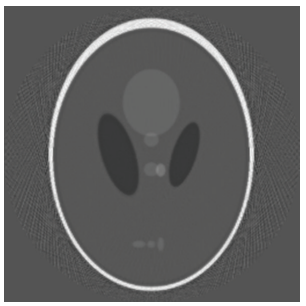

(f)

Figure 2: Original phantom and reconstruction results of the middle plane. (a) is the original phantom of the midplane. (b) is the reconstruction result with the Radon kernel. (c) is the result with the original FDK kernel model. Results with the compact FDK kernel model where the radius $\rho=80,40$, and 4 are shown in (d) (e) (f), respectively.

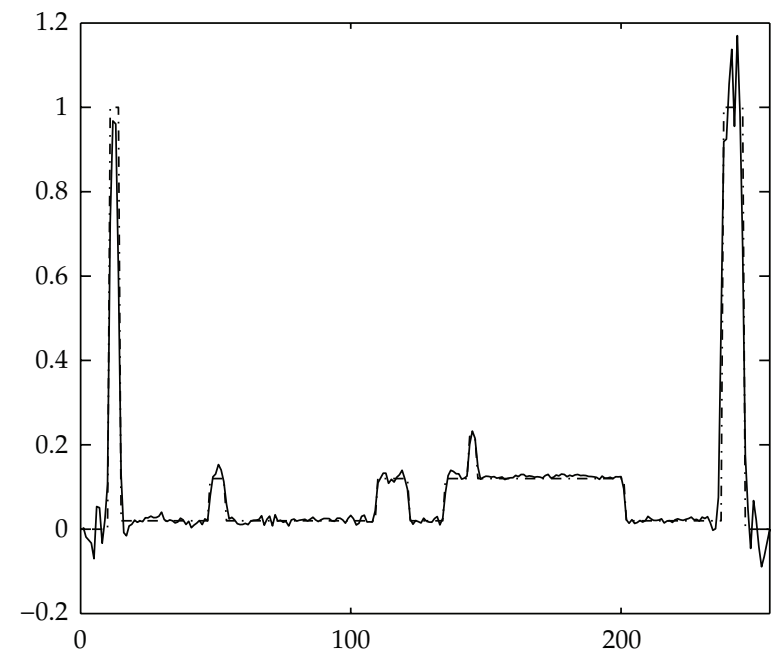

Figure 3: Profiles along the midline of the midplane where the dashed and solid curves represent the true and reconstructed values with the compact FDK kernel with $\rho=4$.

the FDK kernel infinite. The oscillations locate in two vertical directions passing through the center. And at other points, the values are very little as zero. The kernel gives the contribution of the projections around the reconstruction point. In a practical reconstruction work, based on the theory of the approximate inverse, the projections are managed on the kernel with a convolution. These oscillations present the contribution errors in multiple channels. When the projections in the whole circle is managed in the back-projection process, these errors 


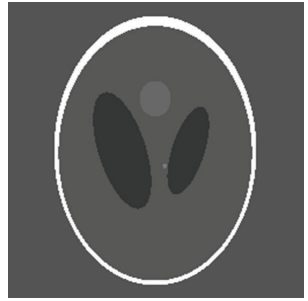

(a)

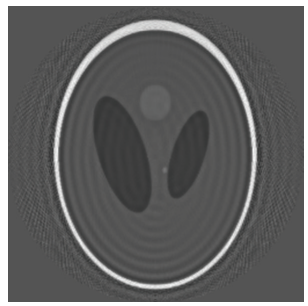

(d)

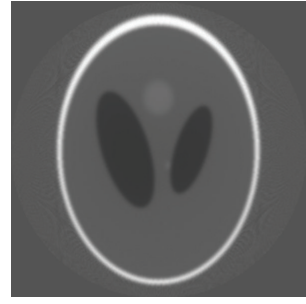

(b)

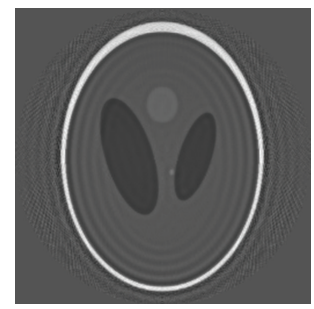

(e)

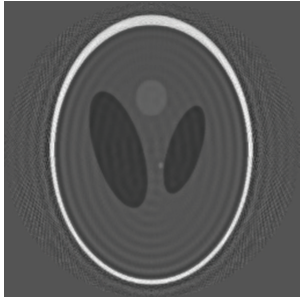

(c)

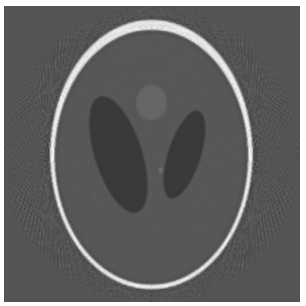

(f)

Figure 4: Original phantom and reconstruction results of the plane parallel and $20 \mathrm{~mm}$ apart from the midplane. (a) is the original phantom of the plane. (b) is the reconstruction result with the Radon kernel. (c) is the result with the original FDK kernel model. Results with the compact FDK kernel model where the radius $\rho=80,40$ and 4 are shown in (d), (e), (f), respectively.

generate the ring artifacts. Especially when reconstructing a point near a high-value area, the value of the point is influenced by the high-value points and these oscillations, and the value is enlarged, which gives rise to the ring artifacts.

In our study, we employ a simple but useful method to reduce the ring artifacts. In order to eliminate the oscillations and make the kernel compact, the kernel is truncated with different radius. A truncation operator $L_{\rho}$ acting on the kernel is present to reach this aim. For a given radius $\rho$, the values of the point $p$ far away from the center $o$ is set to zero, as

$$
\Psi_{\rho}=L_{\rho}(\Psi, p)= \begin{cases}0, & |o p|>\rho \\ \Psi(p), & |o p| \leq \rho .\end{cases}
$$

$p$ is the point on where $\Psi$ is the kernel to be truncated, $|o p|$ is the distance from the center $o$ to the point $p$.

Then projections are processed on the compact kernel for reconstruction according to the approximate inverse method and then we can denote our compact reconstruction model as follows:

$$
f_{\rho}(x)=\left\langle g, \Psi_{\rho}(x)\right\rangle=\left\langle g, L_{\rho} \Psi_{\gamma}(x)\right\rangle
$$

where $f_{\rho}$ is the reconstruction result with the compact kernel, $g$ is the measured projection data.

Results with the compact kernel are also shown in Figures 2(d)-2(f), 4(d)-4(f), and $5(d)-5(f)$. With the compact kernel, the ring artifacts are all eliminated sufficiently. 


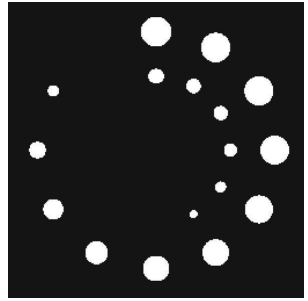

(a)

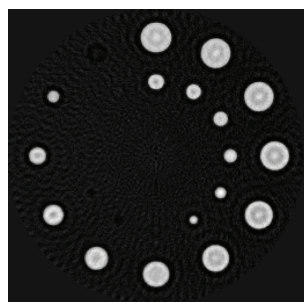

(d)

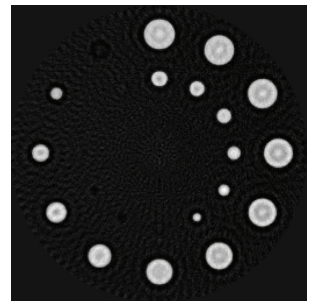

(b)

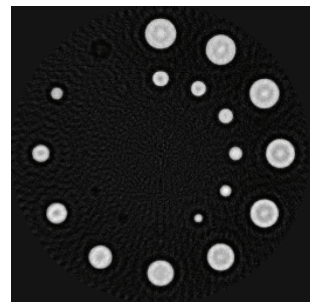

(e)

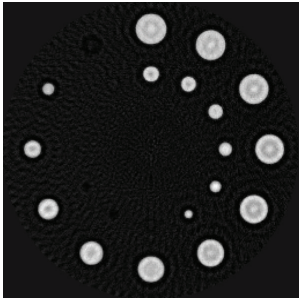

(c)

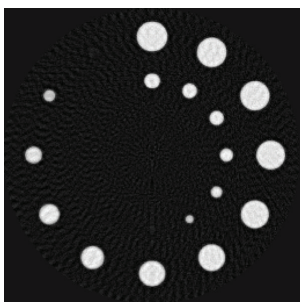

(f)

Figure 5: Original Turbell clock and reconstruction results. (a) is the original Turbell clock. (b) is the reconstruction result with the Radon kernel. (c) is the result with the original FDK kernel model. Results with the compact FDK kernel model where the radius $\rho=80,40$, and 4 are shown in (d), (e) and (f), respectively.

\subsection{Some Invariances of the Kernel}

As the kernel is a function of the reconstruction point $r$, every point may have a different kernel. If the kernel has the invariance property, the efficient of the reconstruction can be improved. In [5], the invariance of the Radon kernel is introduced. Fortunately, the FDK kernel has the property of invariance like the Radon kernel.

From the derivation of the kernel, we can see that the kernel is expressed with the coordinate system on the detector plane. And as coordinate system on the detector plane is shift invariant, the invariance and symmetries of the chose reconstruction relation operator are all directly inherited by the kernel. And with the translational and rotational invariant Gaussian function as the mollifier, the FDK kernel has the invariance below.

For the fixing point $r=0$, from the explicit formula of the operators $M, H, W, D$, and $e_{\gamma}$, for another source point $a^{\prime}$, we can see that these operators are all rotation invariant depending on the local coordinates of the direction $\theta(Y, Z)$. With the unit rotation $I_{M}$ defined as $a^{\prime}=I_{M} a$, the $\theta(Y, Z)$ also rotates to $\theta^{\prime}\left(Y^{\prime}, Z^{\prime}\right)$ coming from $a^{\prime}$ with the relation: $\theta^{\prime}\left(Y^{\prime}, Z^{\prime}\right)=$ $I_{M} \theta(Y, Z)$, then the local coordinates of the points on the kernel keep unchanged, and the kernel keeps unchanged. Thus, we get the following invariance:

$$
\Psi_{\mathrm{FDK}}\left(0, a^{\prime}, \theta(Y, Z)\right)=\Psi_{\mathrm{FDK}}\left(0, a, I_{M}^{T} \theta(Y, Z)\right)
$$

As the position $r \neq 0$ is considered, the direction $\overrightarrow{r a}$ is so close to the direction $\overrightarrow{o a}$. Thus, for simplicity, the corresponding kernel can be represented by the kernel of the position $r=0$ 
with a scale $\left[d^{2}+y^{\prime 2}+z^{\prime 2}\right] / d^{2}$, where $y^{\prime}$ and $z^{\prime}$ are defined in (3.4), then we get the following equation:

$$
\Psi_{\mathrm{FDK}}(r, a, \theta(Y, Z))=\frac{d^{2}+y^{\prime 2}+z^{\prime 2}}{d^{2}} \Psi_{\mathrm{FDK}}(0, a, \theta(Y, Z))
$$

With the above two results, the kernel of an arbitrary point $r$ with the source rotates to $a^{\prime}$ can be represented as

$$
\Psi_{\mathrm{FDK}}\left(r, a^{\prime}, \theta(Y, Z)\right)=\frac{d^{2}+y^{\prime 2}+z^{\prime 2}}{d^{2}} \Psi_{\mathrm{FDK}}\left(0, a, I_{M}^{T} \theta(Y, Z)\right) .
$$

Then for the reconstruction work, we are needed to compute the kernel at the $r=0$ with the fixed source position $a$ for the all directions come from $a$ only once, and the kernels for other $r$ and $a^{\prime}$ can be obtained just with a scale. And these kernels are adopted in the numerical experiments to test our compact FDK kernel reconstruction model.

\section{Numerical Experiments}

In the experiments, the original FDK kernel reconstruction model and the compact FDK kernel reconstruction model are employed for comparison. We choose the well-known Shepp-Logan phantom and the Turbell clock phantom [11] for experiments. Simulation results with these model are shown in Figures 2, 4, and 5. Table 1 shows the reconstruction parameters about the CT geometry. The number of the acquisition views we choose is 180 . More works with other numbers of acquisition views are also carried out, and the same contrast can be getting.

Figure 2 shows the reconstruction results of the midplane of the Shepp-Logan phantom. Figure 2(a) is the original midplane of the Shepp-Logan phantom. For comparison, we reconstruct the phantom with the Radon kernel, and the results are shown in Figure 2(b). Figure 2(c) shows the reconstruction result of the middle plane with the original FDK kernel model. The image is distinct, but many concentric ring artifacts arise in the image, and the edge of the phantom is blurred by the ring artifacts.

Reconstruction results with compact FDK kernel model are shown in Figure 2(d)-2(f) with the radius 80,40 , and 4 , respectively. Different radiuses from nontruncation to 4 are all compared to suppress the ring artifacts. And we present the results with radius 80, 40, and 4 , and the changing of the ring artifacts is obvious. As the ring artifacts are aroused by the oscillations of the FDK kernel, when the radius gets small, the kernel is truncated to be compact, the oscillations are removed, and the ring artifacts are eliminated simultaneously. We can see that with the compact FDK kernel reconstruction model of $\rho=4$, in Figure 2(f), the ring artifacts are suppressed, and the clarity of the image is preserved and not blurred as the truncation is processed. Especially at the edge of the phantom, as the ring artifacts are suppressed, the influence of the ring artifacts is eliminated, and the edge is deblurred. The profiles along the midline of the midplane for the compact FDK kernel reconstruction moedl with $\rho=4$ are shown in Figure 3 .

Figure 4 shows the original phantom and the reconstruction results of the plane apart from the middle plane by $20 \mathrm{~mm}$. Similar details can be seen as Figure 2 . 
Figure 5 is the results of the Turbell clock phantom. The Turbell clock phantom is a high contrast phantom with many sharp density variations in the $z$-direction. Figure 5 shows the original and reconstruction slice of $z=0$. Figure 5(a) is the original Turbell clock. Figure 5(b) is the reconstruction result with the Radon kernel. Figure 5(c) is the result with the original FDK kernel model. Results with the compact FDK kernel model where the radius $\rho=80,40$, and 4 are shown in Figures 5(d), 5(e), and 5(f), respectively.

\section{Conclusion}

In this paper, a novel CT reconstruction model is proposed based on the approximate inverse where the kernel of the FDK method is derived and is used to complete the reconstruction. In order to eliminate the imposed ring artifacts, the kernel is truncated with proper radius. Reconstruction results show that the compact support FDK kernel reconstruction model can suppress the ring artifacts. The proposed reconstruction model preserves the simplicity of the FDK reconstruction method and also provides an alternative to realize the approximate inverse method for circular trajectory. And when the kernel of an algorithm is modified, the corresponding reconstruction formula is also modified accordingly. And this gives us another way to improve the existing reconstruction methods.

\section{Acknowledgment}

This work is supported in part by Scientific Research Program Funded by Shaanxi Provincial Education Department (Program no. 11JK0504).

\section{References}

[1] A. K. Louis and P. Maass, "A mollifier method for linear operator equations of the first kind," Inverse Problems, vol. 6, no. 3, pp. 427-440, 1990.

[2] A. K. Louis, "Approximate inverse for linear and some nonlinear problems," Inverse Problems, vol. 12, no. 2, pp. 175-190, 1996.

[3] A. K. Louis, "Development of algorithms in computerized tomography," in The Radon Transform, Inverse Problems, and Tomography, G. Olafsson and E. T. Quinto, Eds., vol. 63 of Proc. Sympos. Appl. Math., pp. 25-42, Amer. Math. Soc., Providence, RI, USA, 2006.

[4] P. Grangeat, "Mathematical framework of cone beam 3D reconstruction via the first derivative of the Radon transform," in Mathematical Methods in Tomography, vol. 1497 of Lecture Notes in Math., pp. 66-97, Springer, Berlin, Germany, 1991.

[5] A. K. Louis, T. Weber, and D. Theis, "Computing reconstruction kernels for circular 3-D cone beam tomography," IEEE Transactions on Medical Imaging, vol. 27, no. 7, pp. 880-886, 2008.

[6] I. A. Feldkamp, L. C. Davis, and J. W. Kress, "Practical cone-beam algorithm," Journal of the Optical Society of America A, vol. 1, no. 6, pp. 612-619, 1984.

[7] K. Zeng, Z. Chen, L. Zhang, and G. Wang, "An error-reduction-based algorithm for cone-beam computed tomography," Medical Physics, vol. 31, no. 12, pp. 3206-3212, 2004.

[8] D. Soimu, I. Buliev, and N. Pallikarakis, "Studies on circular isocentric cone-beam trajectories for 3D image reconstructions using FDK algorithm," Computerized Medical Imaging and Graphics, vol. 32, no. 3, pp. 210-220, 2008.

[9] Z. Chen and R. Ning, "Volume fusion for two-circular-orbit cone-beam tomography," Applied Optics, vol. 45, no. 23, pp. 5960-5966, 2006.

[10] J. Hsieh, Computed Tomography: Principles, Design, Artifacts, and Recent Advances, Scincep, Beijing, China, 2006.

[11] H. Turbell and P.-E. Danielsson, "Helical cone-beam tomography," International Journal of Imaging Systems and Technology, vol. 11, no. 1, pp. 91-100, 2000. 


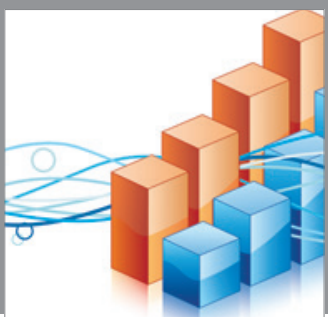

Advances in

Operations Research

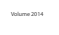

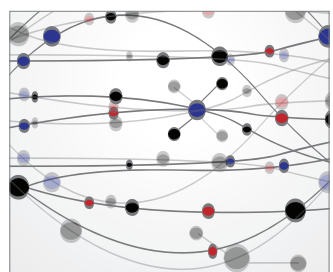

\section{The Scientific} World Journal
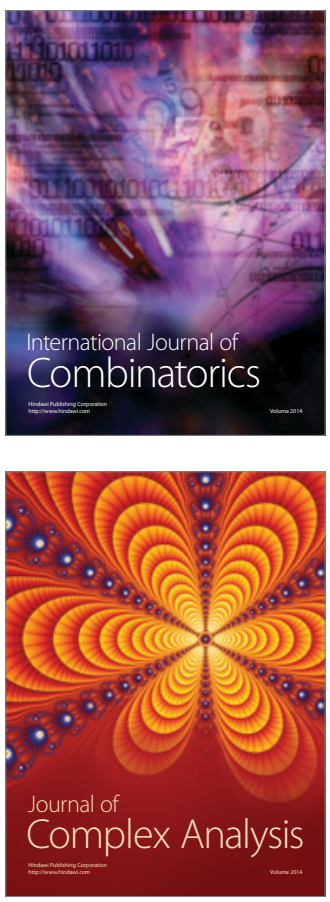

International Journal of

Mathematics and

Mathematical

Sciences
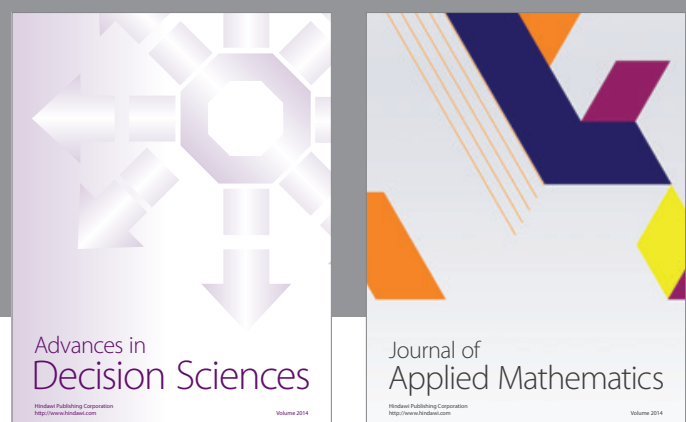

Journal of

Applied Mathematics
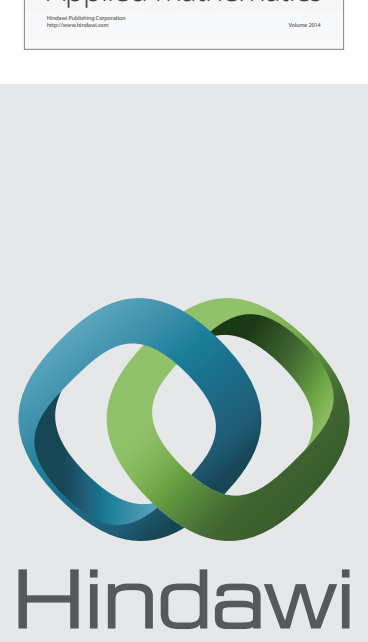

Submit your manuscripts at http://www.hindawi.com
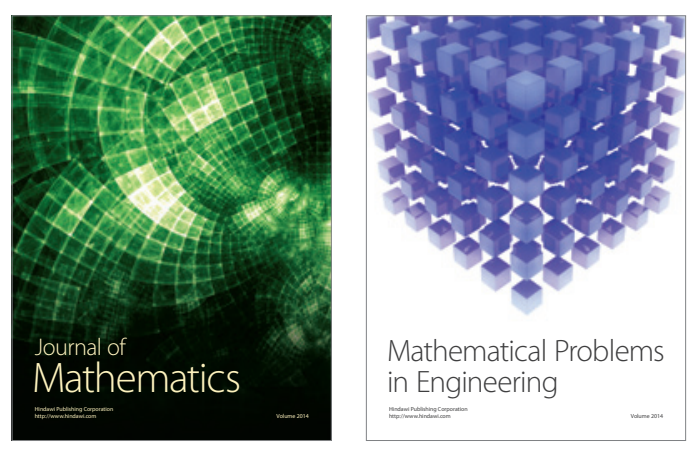

Mathematical Problems in Engineering
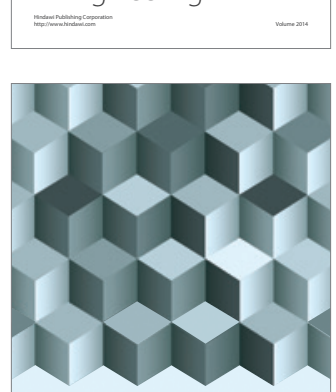

Journal of

Function Spaces
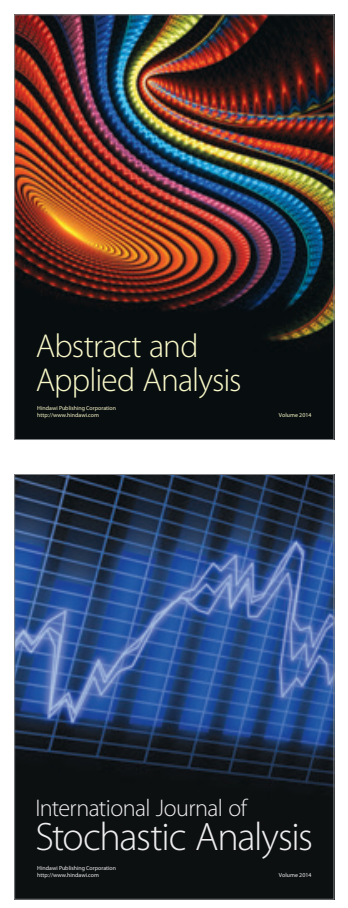

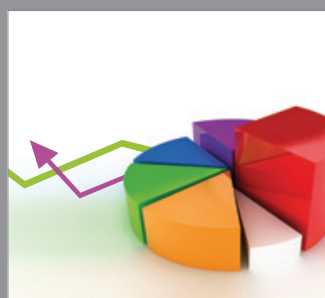

ournal of

Probability and Statistics

Promensencen
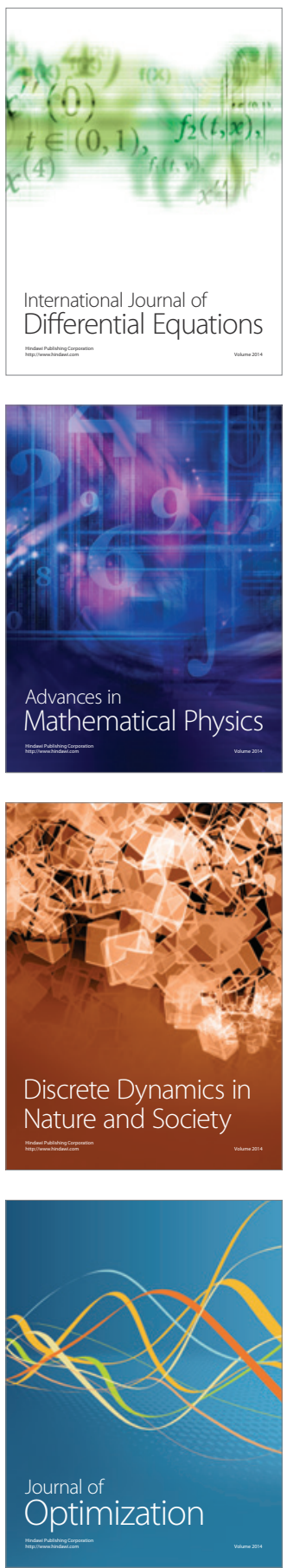